\title{
Parametri non lineari di campi elastici e oscillazioni libere della Terra
}

\author{
(Non linear parameters of elastic fields and Earth's free modes)
}

\author{
M. Caputo - A. Piva (*)
}

Ricevito il 5 Dicembre 3970

Rrassunto. - Questa ricerea d̀ un tentativo di spiegare l'esistenza dolle righe ossorvate nello spettro delle oscillazioni libere della Terra non islentificabili con righe previste per i modelli più attendibili.

Prima si stunlia il comportamento non lineare degli strumenti di misura e la loro interazione col fenoneno; e si vede che questa interazione non potrebbe causare lo righe anomale ma, al più un allargamento delle righo osservate. Poi si studiano i fenomeni non lineari nel caso delle oscillazioni puramente raliali e si vole che por doterminare i parametri elastici che caratterizzano il comportanonto non lineare del campo, sono necessarie molte piu informazioni che per i paranetri lineari; si suggerisec inoltre un metodo per il calcolo di questi parametri eul infue si lisente la possibilita di osservare questi fenomeni nelle oscillazioni libere della Terra.

Summary. - In this paper we attempt to explain the lines observed in the spectrum of the free modes of the Earth which cannot be identified with those of the most probable models.

First we stuxly the non linear behaviour of the instruments and their interaction with the phenomenon; we sec that this interaction cannot cause the anomalous lines, but at most, a thickening of the observed lines. Then we study the non linear phenomena in the purely radial nodos, and we see that, to determine the elastic paramoters which caracterize the non linear behaviour of the field, we noed more information than in the case of the parameters of the linear case; we suggest then a method for the computation of these parameters ant finally, we discuss the possibility to observe these plonomena in the free modes of the Earth.

(*) Istituto di Fisica e Istituto di Geodesia - Zniversila di Bologna. 


\section{1. - INTRODUZIONE.}

T'analisi spettrale delle oscillazioni libere della Terra mostra l'esistejza di righe non identifiabili con quelle previste dalla teoria e che possonto essere dovute alla interazione di dilTerenti modi delle oscillaziout libere, ausate cive da fenomeni nou lineari.

Sebbene vi siano motivi $(1,1)$, primo firb tutti la piccolezza delle deformazioni all interno della Terra, per scartare questa ipotesi, e considerare queste nuove righe spettruli alla stessa stregrua delle altre, i pur sempre valida l'analisi della possibilità di esistenza di questi feunomejli $(2,4)$.

Un altro motivo rhe permettereblje di spiegne la presejta di queste righe, potrebbe essere la perturbazione apportata alle osservazioni dacrli struunenti di misura che potrebbero comportarsi non linearmente. La presente indagine porba ad escludere quest'ultima possibilití e a rousiderare improbabile la prima.

The difficoltà inerenti lo studio dei fenomeni non lineari sono sia fisiche che matematiche. Sorge infatbi il problema della inter]retazione fisica dei fenomest nella regione della soon liuteariti che è resa ancora più diflicoltosa per la rarenza di dati sperimentali. I tentativi finora intræapresi tendono all'emunciazione di leggi fisirhe rhe, al limite dell'ajurossimazioue liueare, si riducono alle legrgi grià note. Questa peró ¿ solo una condizione necessaria ma non sufficiente affinclú una teoria Jon lineare sia considerata valida. Nel rampo della elasticitic della Term, gli spostamenti sone talmente piecoli the mulla si juo ancom dire circa i parametri elastiri dei materiali nella regrione di non linearità, per quanto la teoria qui sviluppata formisca un metodo per il calcolo dei parametri che caratterizanıo al secondo ordine il comportamento di 121 c rampo elastico.

Nella teoria infiutesimale della elastiritì, si fa l'ipotesi the le deformazioni dei corpi siano piccole. Questo significa che la variazione delle distanze è piccoln rispetto alle distumze stesse; in altri termini gli allungamenti relativi souto tali che:

$$
\frac{d l^{2}-d l^{2} o_{o}}{d l^{2}} \ll 1
$$

Iu bal caso, butte le romponenti del tensore di deformazione che determinano le variazioni relative di lomglıeza, soso pure piceole rispetto all'unità. 
In base a queste ipotesi, i fenomeni elastici sono regolati essenzialmente dalla legge di Ilooke che stabilisce la proporzionalità fia il tensore degrli sforzi e quello delle deformazioni. Quando le deformazioni dei rorpi non si possono più considerare piccole, la legge di Hooke nom è più valida essendo neressario tenere in considerazione anche i termini di ordine superiore al primo nelle componenti clel tensore di deformazione.

Le difficoltà di ordine matematico consistono essenzialmente nel fatto die le equazioni del moto, essendo non lineari, non godono del principio di sovmaposizione. Ie soluzioni di queste equazioni contengono delle funzioni ale descrivono il comportamento cosiddetto anarmonico del mezzo elastico e sono cantterizate dalie frequenze di rombinuzione.

Ja teoria delle deformazioni finite svilupjata dal Murnaghan (5) ¿̀ uno sviluppo rigoroso della teoria della elasticità in cui non si impone aleuıa restrizione alla grandezza della deformazione. Isa teoria ì formalmente esatta, ma, in pratica, sorgono delle limitazionj poiché nulla si sa sui valori dei coeflicienti di ordine superiore al serondo the ronpaiono nello sviluppo della energia di deformazione in fumzione della deformazione stessa.

Come si vede, la grandezza ele stabilisce se un mezzo elastico anmette o no un comportamento non lineare i la deformazione del mezzo stesso. Per la Terra il suo valore $\grave{e} \simeq 10^{-8} \mathrm{e}$, di consegruenza, al secondo ordine is cosi piccolo che difficimente potrà fornire al termine non lineare delle oscillazioni libere n'ampiezza osservabile.

In Tab. I sono elencati alcumi modi relativi ai fenomeni sismici registrati durante il terremoto del Cile del 1900 e dell'Alaska del 1964, tra i quali ve ne sono alenni per i quali is stato suggerito un fenomeno di interazione. MIa, a nostro avviso, le ampiezze sono troppo piccole per poter pensare che si possa osservarle.

Un esempio ne è il modo ${ }_{1} S_{0}$, la rui frequenza è pressoché doppia di quella dell'armonic:a fondamentale $0 S_{0}$ e che quindi potrebbe essere interpretato come interazione dell'armonica fondamestale con se stessa, ina, dall'aualisi spettrale si puó vedere che il rapporto fra le relative ampiezze osservate sirebbe:

$$
\frac{A\left({ }_{1} S_{0}\right)}{A\left({ }_{0} S_{0}\right)} \simeq \frac{-1}{20}
$$

che, a nostro avviso, è incompatibile con la piccolezza della deforma- 
zione. Questo ai porta a conchudere che il periodo di cires 10 minuti è veramente ${ }_{1} S_{0}$.

TAB. 1

\begin{tabular}{|c|c|c|c|}
\hline \multicolumn{2}{|c|}{ M $0 \mathrm{~d} \mathrm{i}$} & \multirow{2}{*}{$\begin{array}{c}\text { Perioli osservati } \\
\text { nel (bile (min.) }\end{array}$} & \multirow{2}{*}{$\begin{array}{l}\text { Periodi osservati } \\
\text { noll'Alaska (min.) }\end{array}$} \\
\hline Naturali & Interazione & & \\
\hline${ }_{1} S_{0}$ & & 10.016 & 10.22 \\
\hline${ }_{0} S_{B}$ & & 11.80 & 11.89 \\
\hline${ }_{2} S_{5}^{\prime}$ & & $11,00)$ & $11.10-10.98$ \\
\hline 6.5 & & 9.67 & 9.68 \\
\hline \multirow[t]{6}{*}{${ }_{0} K_{11}$} & & 8.93 & 8.95 \\
\hline & ${ }_{0} S_{8}-{ }_{2} S_{5}$ & 152 & 149 \\
\hline & ${ }_{2} S_{5}-{ }_{1} S_{0}$ & 106 & 103 \\
\hline & ${ }_{2} S_{5}-{ }_{0} S_{10}$ & 79 & $79-80$ \\
\hline & ${ }_{0} S_{8}-{ }_{1} S_{0}$ & fil & 61 \\
\hline & ${ }_{2} S_{5}-{ }_{0} S_{11}$ & 48 & $47-48$ \\
\hline
\end{tabular}

2. - MODELII ELASTICI DEGLI STRUMENTJ DI MISURA.

Ci proponiamo ora di esaminare l'interazione Terra-strumento di misurn supponendo che quest'ultimo stmmetta un comportamento non line:zre.

(Gli strumenti che permettono di mettere in evidenza le oscillazioni libere della Tena, che ammettono periodi molto lunghi, sono spesso i gravimetri, o strumenti ad essi equiparabili.

Tali strumenti si possono schematizzare come uni massi, collegata al supporto rigido dello stumento mediante un sistema elastico che in genernle ì nna molla. Quindi, lo studio di questi strumenti di misura si riduce allo studio di oscillatori per i quali, nel caso attuale, si con- 
sidera un termine al secondo ordine jello spostamento nell'espressione della forza elastica di richiamo.

Se il moto del supporto d̀ dellil forma:

$$
s(t)=a_{1} \cos \omega_{1} t+a_{2} \cos \omega_{2} t
$$

l'equazione del moto della massa del grivimetro è data dalla:

$$
m y=-K(y-s)+\lambda(y-s)^{2}
$$

essenzo y lo spostamento dalli posizione di equilibrio, con le coucizioni iniziali: $y(o)=b, y(o)=0$.

Introduceudo una nuova variabile: $x(t)=y(t)-s(t)$, l'equazione del moto divesttis:

$$
\ddot{x}+\omega^{2} 0_{0} x=\alpha^{2} x^{2}+\omega^{2} a_{1} \cos \omega_{1} t+\omega^{2} a_{2} \cos \omega_{2} t, \quad \alpha^{2}=\frac{2}{m}
$$

$a^{2}$ essendo il coefficiente di nom linearità, e ponendo:

$\omega^{2} a_{1}=b_{1}$ e $\omega_{2}^{2} a_{2}=b_{2}$, si ha l'equizione del moto:

$$
\left.\ddot{x}+b^{2}\right)_{0} x-\alpha^{2} x^{2}=b_{1} \cos \omega_{1} t+b_{2} \cos \alpha \cdot t
$$

risolvibile mediante il metodo operativo introducendo la trasformata di Latplace.

Applicando la soluzione trovata allo studio delle oscillazioni libere della Terra, eccitate dal terremoto dell'Alaska nel Maggio 1964, registrate a Los Angeles col gravimetro Lit Coste il eui periodo proprio è di cirra 50 sec., coussideriuddo ad esempio il periodo delle oscillazioni radiali della Terra che d̀ di circa 1200 sec, il termine che ci descrive il comportamento del sistema in seconda approssimazione $\grave{e}$ :

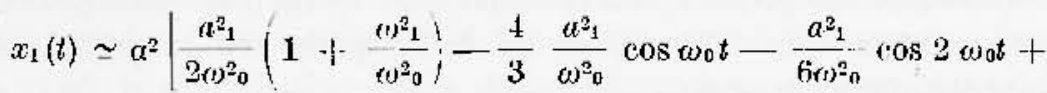

$$
\begin{aligned}
& +\frac{a^{2}}{\left.2 \omega^{2}\right)_{0}}\left(\frac{\omega_{1}}{\omega_{0}}\right)^{2} \cos 2 \omega_{1} t+a^{2}\left(\frac{\omega_{1}}{\omega_{0}}\right)^{2} \frac{\cos \left(\omega_{0}+\omega_{1}\right) t}{\omega^{2}{ }_{1}+2 \omega_{0} \omega_{1}}+ \\
& \left.+a^{2}\left(\frac{w_{1}}{w_{0}}\right)^{2} \frac{\cos \left(\omega_{0}-\omega_{1}\right) t}{\omega^{2}-2 \omega_{0} \omega_{1}}\right]
\end{aligned}
$$

dove le nuove frequueuze $2 \omega_{0}, \omega_{0} \pm \omega_{1}$ e $2 \omega_{1}$ ei inddicano rispettivamente i seguenti tipi di iuterizione: 
1) Interazione dello strumento di misura $\omega n$ se stesso: per la magrior parte degli strumenti con i quali si sono osservate le oscillazioni libere della Terra fino ad oggi è $\omega_{0} \leqslant 50^{-1}$ eps. per rui la frequesza $2 a_{0}$ non introduce alenu problema nell'analisi delle frequeruze proprie della Terra;

2) Interazione Terra-strumento di misura.

Le frequenze:

$$
\omega_{0} \pm \omega_{1}=\omega_{0}\left(1 \pm \frac{\omega_{1}}{\omega_{0}}\right) \simeq \frac{1}{50}\left(1 \pm \frac{1}{24}\right)
$$

corrispondono a periodi che sono minori di 1 min. e poiche le letture delle registrazioni vengono fatte a intervalli di 1 min. la loro eventuale presenza c un evento aleatorio.

3) Interazione Terra-Terra.

Nel caso della oscillazione ${ }_{0} S_{0}$, la rorrispondente frequenza $\left(m_{1}\right.$ da luogo ad una frequenza $2 \omega_{1}$ ohe $i$ quasi identica alla prima armonica di oSo che è ${ }_{0} S_{0}$; l'ampiezza del termine in $2 \omega_{1}$ è peró $\simeq 3.7 \cdot 10^{-3} q^{*} \alpha^{2}{ }_{1}$ ciò tale da non destare alcm problema nell'analisi dei dati poirhé $a_{1}<1 \mathrm{~cm}$ ed il coefficente $a^{-}$è molto piccolo.

Sembra quindi che un eventuale comportamento nou lineare degli strumenti di misum non spieghi la presenza delle righe anomale.

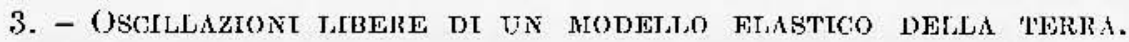

Passiamo ora allo studio delle oscillazioni libere di ura sfera elastica omogenea, di densità $\varrho$, raggio $R$ e parametri elastici $\lambda$ e $\mu$ allo seopo di indirare la possibilitì di determinare sperimentalinente i coeffucienti elastici di non linearità, estendendo lo studio delie oscillazioni libere alla seconda aprossimazione.

Introduciamo prima alcuni richiami sulla teoria delle deformazioni finite enunciata dal Mumaglan (5) dalla quale siamo partiti per sviluppare questo luvoro.

3.1 - Defnizione della deformazione e costanti dastiohe di un mezzo isotropo.

Nella teoria delle deformazioni finite (5) la matrice di deformazione viene definita nel modo seguente:

$$
\eta=\frac{1}{2}\left(J^{*} J-I\right)
$$


dove: $I$ ì la matrice Jacobiana, relativa alla trasformazione dalle coordinate iniziali $(a, b, c)$ di ma particella del mezzo deformabile alle coordinate finali $(x, y, z)$ della stessa particella, mentre $I$ è la matrice unitaria.

L'energia di deformazione per uuità iniziale di volume viene espressa mediante uno sviluppo in serie di potenze nei tre invarianti $I_{1}, I_{2}$ e $I_{3}$ della matrice di deformazione che, se $\grave{e}$ scritta in forma ("anonica, assumono la forma:

$$
\begin{aligned}
& I_{1}=\eta_{1}+\eta_{2}+\eta_{3} \\
& I_{2}=\eta_{1} \eta_{2}+\eta_{1} \eta_{3}+\eta_{2} \eta_{3} \\
& I_{3}=\eta_{1} \eta_{2} \eta_{3}
\end{aligned}
$$

mentre l'energia di deformazione si può scrivere:

$$
\phi=\phi_{1}+\phi_{2}-1-\phi_{3}+\ldots
$$

essendo:

$$
\begin{aligned}
& \phi_{1}=\alpha I_{1} \\
& \phi_{\mathrm{a}}=\frac{\lambda+2 \mu}{2} I_{1}{ }_{1}-2 \mu I_{2} \\
& \phi_{\mathrm{a}}=\frac{l+2 m}{s} I_{1}-2 m I_{1} I_{2}+m I_{3}
\end{aligned}
$$

dove i parametri $\lambda, u, l, m, n$ sono le costanti elastiche del mezzo isotropo.

Passiamo ora allo studio del problema annunciato: consideriamo una particella del mezzo (-he abbia, allo stato iniziale, coordinate $(r, \hat{v}, \varphi) \cdot$ la sua posizione nello stato deformato sarà individuata dalle coordinate $(r+h u, \vartheta, \varphi)$ dove $k u$ rappresenta lo spostamento.

La rostante $h$ è adimensionale ed è tale rhe nella teoria lineare trascuriano le sue potenze superiori alla prima, mentre nella seconda approssimazione ne trascuriamo le potenze superiori alla seconda.

Le oscillazioni radiali sono caratterizzate da:

$$
u-u(r, t), u \vartheta=u \varphi \equiv 0 .
$$


Supponendo per semplicità cle il mezzo sia libero da stress iniziale, la funzione densità di energia è espressa dalla formula:

$$
\phi=\frac{2+2}{2}-I_{1}^{2}-2 \mu I_{2}+\frac{l+2 m}{3} I_{1}^{3}-2 m I_{1} I_{2}+n I_{3}
$$

dove, trascurando le potenze di $k$ superiori alla seconda, abbiamo:

$$
\begin{gathered}
I_{1}=k\left(u^{\prime}+\frac{2 u}{r}\right)+\frac{1}{2} \bar{k}^{2}\left(u^{\prime}+\frac{2 u^{2}}{r^{2}}\right) \\
I_{2}=k^{2}\left(\frac{u^{2}}{r^{2}}+\frac{2 u u^{\prime}}{r}\right) .
\end{gathered}
$$

La matrice di stress è:

$$
T=J \frac{\partial \phi}{\partial \eta}
$$

dove:

$$
\frac{\partial \phi}{\partial \eta}=\lambda I_{1} I+2 \mu \eta+\left(I I_{1}^{2}-2 m I_{2}\right) I+2 m I_{1} \eta+n c o \eta
$$

L'equazione del moto in forma matriciale, in assenza di forze di volume è:

$$
(\operatorname{div} T)^{*}=\varrho x_{\iota t}
$$

Esplicitumente si ha:

$$
\frac{\partial T_{r r}}{\partial r}+\frac{2}{r}\left(T_{r r}-T \vartheta \theta\right)=\varrho(k u)
$$

Poneuto:

$$
u(r, t)=u_{1}(r, t)+k w(r, t)
$$

dove $u_{1}(r, t)$ ci rappresenta la soluzione del problema in prima approssimazione e $w(r, b)$ la correzione in seconda approssimazione, l'equazione del moto in prima approssimazione è:

$$
\varrho \ddot{u}_{1}=(\lambda+2 \mu)\left[\frac{\partial^{2} u_{1}}{\partial r^{3}}+\frac{2}{r} \frac{\partial u_{1}}{\partial r}--\frac{2 u_{1}}{r^{2}}\right] ; \frac{\lambda+2 \mu}{\varrho}=V^{2}{ }_{l}
$$

dove $V_{\imath}$ è Ia velocitì longitudinale di propagazione delle onde elastiche. 
Cerchiamo la soluzione di [3.13] nella classe:

$$
u_{1}(r, t)=u_{0}(r) e^{i \omega_{1} t}
$$

dove $u_{0}(r)$ sodilisfit l'equazione:

$$
\frac{d}{d r}\left|\frac{1}{r^{2}} \frac{d}{d r}\left(r^{2} n_{n}\right)\right|+h^{2} x_{0}=0
$$

ron $k_{1}=\alpha h_{1} / V_{t}$ vettore d'omilit.

La soluzione finita all'origine $i$ :

$$
u_{0}(r)=A\left(\frac{\operatorname{siu} k_{1} r}{\left(k_{1} r\right)^{2}}-\frac{\cos k_{1} r}{k_{1} r}\right)=c_{1} \frac{J_{3 / 2}\left(k_{1} r\right)}{\sqrt{r}}
$$

La condizione al contocus:

$$
T_{r r}=0 \text { per } r=K
$$

fornisce gli autovalori $k_{1}$ jer i quali $i$ valida la soluzione e che soddisfauo la ben nota relazione:

$$
\frac{t y k_{1} R}{k_{1} R}=\frac{1}{1-\left(\frac{\lambda+2 \mu}{4 \mu}\right)\left(k_{1} R\right)^{2}}
$$

In serondit approssimazione, trascurando le potenze in $k$ superiori alla sesonda nel alcolo dolle compoutenti della matrice di sforzo $T$, derivando, racogliendo e separando i termini in $k$ e $k^{2}$ si ottiene lequazione giò seritta in presedenza e che di dì la soluzione al primo ordine $u_{1}(r, t)$ ed inoltre l'equazione segnente ottenuta ammullando i coefficienti di $k^{2}$ e trascurando $\mathrm{i}$ termini relativi alle potenze superiori:

$$
\begin{gathered}
\frac{\partial^{2} w}{\partial t^{2}}-r_{i} \frac{\partial}{\partial r}\left[\frac{1}{r^{2}} \frac{\partial}{\partial r}\left(r^{2} w\right)\right]=\frac{\lambda+2 l}{0} \dot{i}_{u 1} \frac{\partial A_{u 1}}{\partial r}+ \\
+\frac{2(\lambda+3 \mu+2 m)}{\varrho} \frac{\partial u_{1}}{\partial r} \frac{\partial A_{u}}{\partial r}- \\
-\frac{2(\lambda+3 \mu+2 m)}{g} \frac{1}{r}\left(\frac{\partial u_{1}}{\partial r}-\frac{u_{1}}{r}\right)^{2} \\
\Delta u_{1}=\frac{\partial u_{1}}{\partial r}+\frac{2 u_{1}}{r}
\end{gathered}
$$


Ponendo:

$$
\frac{\lambda \cdot 2 l}{\varrho}=\alpha^{2}, \frac{2(\lambda-3 \mu \div 2 m)}{\varrho}=\beta^{2}
$$

sicooune:

$$
\frac{\partial \lambda u_{1}}{\partial r}=\frac{1}{v_{i} r} u_{1}
$$

l'equazione si può serivere cosi:

$$
\begin{gathered}
\left.\frac{\partial^{2} w}{\partial t^{2}}-V_{t^{2}} \frac{\partial}{\partial r}\left|\frac{1}{r^{2}} \frac{\partial}{\partial r}\left(r^{2} w\right)\right|=-\mid \frac{\left(u^{2} \alpha^{2}\right.}{V_{t^{2}}}\left(\frac{d u_{0}}{d r}+\frac{2}{r}\right) u_{0}\right) u_{0}+ \\
+\frac{\omega_{1} \beta^{2}}{V_{t}^{2}} \cdot u_{0} \frac{d u_{0}}{d r}+\beta^{2} \frac{1}{r}\left(\frac{d u_{0}}{d r}-\frac{n_{0}}{r}\right)^{2} \mid e^{i \omega_{1} t}
\end{gathered}
$$

questa equazione, alie derivate parziali, nom omogenen, is di difficile soluzione; per ora cerchiamo di ottenere una soluzione mediante separazione della variabili, ma sarà oggretto di ricerche ulteriori la soluzione del nostro problema alottando un metorlo diverso. Consideriamo [erciò uza soluziouse del tipo:

$$
w(r, t)=w_{0}(r) e^{i \omega_{2} t}
$$

si hal :

$$
\begin{aligned}
& \left|\frac{d-w_{0}}{d r^{2}}+\frac{2}{r} \frac{d w_{0}}{d r}+\left(\frac{\left.\sigma^{2}\right)^{2}}{V_{t^{2}}}-\frac{2}{r^{2}}\right)\right| e^{2} \omega_{2} t= \\
& =\mid \frac{k_{1}^{2} \alpha^{2}}{V_{2}^{2}}\left(\frac{d u_{0}}{d r} u_{0}+\frac{2 u_{0}}{r}\right) u_{0}+\frac{l^{2} \beta^{2}}{V_{t}^{2}} \cdot u_{0} \frac{d u_{0}}{d r}+ \\
& +\frac{\beta^{2}}{l_{t}^{2}} \frac{1}{r}\left(\frac{d u_{0}}{d r}-\frac{u_{0}}{r}\right)^{2} \mid e^{i 2 \omega_{1} t} .
\end{aligned}
$$

Questa i uma identità nel tempo e pró essere soldisfatta ponendo:

$$
\omega_{2}=2 \omega_{1}
$$


C'io implisa che le oxrillazioni libere in seconda approssimazione comportano delle frequenze alus sono doppie di quelle relative alla approssimazione lineare. Poniamo:

$$
\frac{w^{2}}{r_{t^{2}}^{2}}=k^{2}{ }^{2}
$$

Possiamo quindi serivere l'equazione del moto nella forma:

$$
\begin{aligned}
& \frac{d-w_{0}}{d r^{2}}+\frac{2}{r} \frac{d w_{0}}{d r}+\left(k_{2}-\frac{2}{r^{2}}\right) w_{0}=\frac{c^{2} \beta^{2}}{V_{t^{2}}} \frac{1}{r^{2}}\left(\frac{d J}{d r}\right)^{2}+ \\
& +\sigma_{1}^{2}\left[\frac{\left(a^{2}+\beta^{2}\right) k^{2}{ }_{1}}{V_{t^{2}} r}-\frac{3 \beta^{2}}{V_{t^{2} \beta^{2}}^{2}} \mid J \cdot \frac{d J}{d r}+\right. \\
& +e^{2}{ }_{1}\left\lfloor\frac{\left(3 \alpha^{2}-\beta^{2}\right) k^{2}}{2 V_{l^{2}} r^{2}}+\frac{9}{4} \frac{\beta^{2}}{V_{t^{2} \gamma^{-1}}}\right\rfloor \ldots J^{2}
\end{aligned}
$$

dove $J$ é la funzione di Ressel:

$$
I_{3 / 2}(k, r)
$$

Ponendio:

$$
B_{1}=\frac{c^{2} \alpha^{2}}{V_{l^{2}}}, \quad B_{2}=\frac{c^{2} \beta^{2}}{V_{t^{2}}}
$$

rerbhiamo ora una soluzione dellecquzione nel raso che i parametri siano tali da soddisfare la seguente relazione:

$$
B_{1}=-B_{2} \text { (iò̀ } \alpha^{2}=-\beta^{2}
$$

ovvero:

$$
3(\lambda+2 \mu)+2 l+1 m=0 .
$$

Il che equivale ad adottare un particolare modello elastico della terra.

In tal caso la soluzione finita nell'origine è:

$$
w_{0}(r)=D\left|\frac{\sin 2 k_{1} r}{\left(2 k_{1} r\right)^{2}}-\frac{\cos 2 k_{1} r}{2 k_{1} r}\right|+\frac{5}{8} \frac{B_{1}}{r^{2}} J^{2}+\frac{3}{4} \frac{B_{1}}{r}, \cdot \cdot \frac{d J}{d r} .
$$




\section{2. - Rieorca delle frequenze proprie.}

Consideriamo ancora la condizione al contorno:

$$
T_{r r}-0 \quad \text { per } \quad r=R
$$

che diventa, considerando anche $\mathrm{i}$ termini al serondo ordine:

$$
\begin{aligned}
& { }_{\{}\left\{\lambda\left(u^{\prime}+\frac{2 u}{r}\right)+2 \mu u^{\prime}\right\}+k \cdot \lambda\left(\frac{3}{2} u^{\prime 2}+\frac{2 u u^{\prime}}{r}+\frac{u^{2}}{r^{2}}\right)-1 \\
& \left.+3 \mu u^{\prime 2}+l\left(u^{\prime}+\frac{2 u}{r}\right)^{2}+2 m\left(u^{\prime 2}-\frac{u^{2}}{r^{\prime}}\right)+n \frac{u^{-}}{r^{2}}\right\}=0
\end{aligned}
$$

dove:

$$
u(r, t)-w_{0}(r) i \omega_{1} t-f-h w_{0}(r) r^{i 2 \omega_{1} t}
$$

Se ricordiamo la formula [3.21] e l'espressione di $u_{0}(r)$, la condizione a] contorno al II ordine diventa:

$$
\begin{aligned}
& \frac{t g 2 k_{1} k}{2 k_{1} k}=\frac{1}{1-\left(\frac{\lambda+2 \mu}{+\mu}\right)\left(2 k_{1} R\right)^{2}} \mid 1+ \\
& \quad+\psi(\lambda, \mu, l, m, n) \frac{\pi A^{2}}{D} \cdot \frac{J}{R \cos 2 k_{1} k} \mid
\end{aligned}
$$

ove:

$$
\psi(\lambda, \mu, l, m, n)=\frac{2 l-2 m+n}{1 \mu}-\frac{3 \lambda(\lambda+2 l)(\lambda+6 \mu)}{8 \mu(\lambda+2 \mu)^{2}} .
$$

Se le oscillaziont libere sono tali due la deformazione supera un certo valore, oltre il quale e rilevabile il comportamento non lineare, la relazione precedente ri permette di ricavare informazioni sui coeffirienti di non linearità $l, m$ eil $n$, oltre rhe sulla costante del moto $D$. I in questo senso (lle deve venire interpretata la condizione al contorno relativa alle frequenze dei modi liberi in seconda approssimazione poiclú la teoria wi dice grià whe tali frequenze devono essere doppia di quelle relative alla jrima ipjorossimazione. 


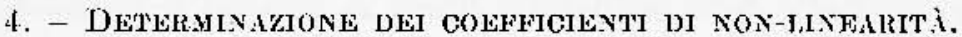

Per la determinazione di tutti e tre i parametri, somo neressarie ameno tre relazioni indipendenti. Infatti, mentre nel caso lineare le misure della frequenza e dell'ampiezza dello spostrmento i sufficiente a definire il romportamento dinamio del sistema, questo non i possibile in seconda approssimazione. Un metodo da seguire per eliminare tale indeterminazione sarà quello di trovare le relazioni whe discendono dall'internzione dell'amonica fondamentale con se stessa e da quella di due altre armoniclie sepanatamente.

Vediamo, mediante un'analisi semi-cuuntitativa, se ì possibile determinare, e sotto quali condizioni sperimentali, i tre roefficienti inrogniti mediante le relazioni trovate.

A questo scopo, oroure fare l'ipotesi muionerole che la costante adimensionale $k$ sia indipendente dalla frequenza.

Consideriamo le espressioni:

$k\left[D_{t} f_{2}\left(\lambda, \mu, \varrho, R, \lambda_{i}\right)+f_{3}\left(\lambda, \mu, l, \varrho, R, h_{i}\right)\right]=k\left({ }^{\prime}\left(\lambda, \mu, l, \varrho, R, R_{i}\right)=\Lambda_{i}\right.$

$$
\begin{gathered}
\frac{t_{0} 2 k_{i} R}{2 k_{i} R}=\frac{1}{1-\left(\frac{\lambda+2 \mu}{4 \mu}\right)\left(2 k_{i} R\right)^{2}} \mid 1+ \\
\left.\quad+\psi(\lambda, \mu, l, m, n) \frac{\pi A^{2} i}{D_{i}} \frac{J^{2} 3 / 2\left(k_{i} R\right)}{R \cos 2 k_{i} R}\right] \\
3 \lambda+6 \mu+2 l+4 m=0, \quad i=0,1,2\left(^{*}\right)
\end{gathered}
$$

dove:

$$
\begin{aligned}
& f_{2}=\frac{\sin 2 k_{i} R}{\left(2 k_{i} R\right)^{2}}-\frac{\cos 2 k_{i} R}{2 k_{i} R} \\
& f_{3}=\frac{5}{8} \frac{B_{1}}{R^{2}} J^{2}{ }_{3 / 2}\left(k_{i} R\right)+\frac{3}{4} \frac{B_{1}}{R} J_{3 / 2}\left(k_{i} R\right) \cdot\left[\frac{d J_{3 / 2}}{d r}\left(k_{i} r\right)\right]_{r=R,}
\end{aligned}
$$

(*) Gli indici (t, I, 2 si riforiseono rispettivaneute alle armoniche: foudamentales, prima o secoura. 
mentre $D_{i}$ è la costaute del moto iucognita della soluzione [3.22]. Jall'analisi spettrale delle oscillazioni libere si possono misurare le frequenze dell'armonica fondamentale e della prima e seconda amonica.

Le misure delie rispettive ampiezze $A_{\text {or }}$ degli spostamenti al primo ordine ri permettono di calcolare, tramite le relazioni:

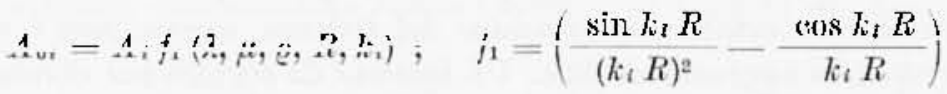

le tre costanti $A_{\mathrm{u}}, A_{1} \mathrm{e} A_{\mathrm{n}}$.

Jisumando per le stesse amouiche le ampiezze degli spostamenti al secondo ordine $\Lambda_{0}, A_{1}$ e $\Lambda_{2}$ si possono ottenere i rapporti:

$$
\begin{aligned}
& E_{1}=\frac{\Lambda_{0}}{\Lambda_{2}} \\
& E_{2}=\frac{\Lambda_{0}}{\Lambda_{2}}
\end{aligned}
$$

dai quali, essendo noti i valori di $\lambda, \mu, 0, R, k_{0}, k_{1}$ e $k$, si possono ricavare le setruenti relazioni:

$$
\begin{aligned}
& D_{0}=D_{0}\left(D_{2}, l\right) \\
& D_{\mathbf{l}}=D_{1}\left(D_{2}, l\right) .
\end{aligned}
$$

Dalle coudizioni al contorno [4.2] possiamo ottenere tre espressioni del tipo:

$$
\phi_{i}\left(D_{2}, l, m, n\right)=0 \quad i=0,1,2
$$

rhe, con la relazione lineare:

$$
\text { 37. }+6 \mu+2 l+4 m=0
$$

determinano un sistema di quattro equazioni nelle quattro incognite:

$$
D_{2}, l, m, n \text {. }
$$


La soluzione di questo sistema ri permette poi di risalire alle rostanti $D_{0}, D_{1}$ e quindi alla costante adimensionale $k$ whe gode della seguente proprietia:

$$
\therefore-\frac{A_{0}}{C_{0}}=\frac{A_{1}}{C_{1}}=\frac{\Lambda_{2}}{C_{2}} \text {. }
$$

Riassumendo si vede che l'estensione dello studio delle oscillazioni libere alla seconda approssimazione richiede la introduziome di maggiori dati sperinentali clu in prima approssimazione.

La misura delle frequenze e delle ampiezze degli spostamonti al primo ordine ci permette di calcolare le costanti $A_{1}(i=0,1,2)$ mentre la misura delle ampiezze degli spostamenti al secouto ordiue permette di ricavare, tramite le relazioni soritte sopra, le rostanti del moto $k, D_{i}$ ed elastiche $l, m$ ed $n$.

Naturalmente, le difficoltà sperimentali sono notevoli poiché la misura degli spostamenti al secoudo ordine riclipde una eccezionale acrurateza nelle misure. Prendendo in considerazione, rome suggerito, l'amonica foudamentale e la prima e la kerouda armonica:

$$
\text { WS, } S_{0} S_{0,2} S_{0}
$$

di periorli rispettivamente di $20.47 \mathrm{mish}, 10.04 \mathrm{~min}$. e $6.60 \mathrm{misl}$. data la limitatezan della deformazione per la Terra, clue implica un'ampiezza dello spostamento molto piccolat, probabilmente solo l'internzioue dellamonion fondanentale cou se stessa potrà essere messa in evidenza a rausa del rumore di fouto.

Il fatto clue le ampiezze degli spostamenti al secoudo ordine siano diffrcilmente distingrubili dal rumore di fouslo, pono delle notovoli limitazioni sperimentali per la determinazione dei corfficienti di nomlinearità.

Questo lavoro serve a descrivere grli aspotti fondamentali dei modelli nou lineari nel ampo dell'olasticiti. Cua teoria più rompleta potrà facilmente tentere routo, oltre che di uno stress iniziale di tipo idrostatico, anche della pariazione del campo gravitazionale rou la deformazione. Il uon tenore ronto di questi fattori, nella teoria lineare, nou cambia la strutturi formale delle soluzioni, e probabilmente questo vale anche in secouda approssimazione. Commoue, queste perturbazioni nou alterano le curatteristiche fondamentali del sistema in secomila approssimazione. 
Per quauto riguarda laspetto matematico della teorin e più precisamejte la soluzioute di equazioni diferenziali nou lineari alle derivate parziali, si è visto che, adottando il metorlo delle approssimazioni sucessive è sempre possibile ridursi al waso di equazioni difterenziali non omogenee alle derivate totali. In difficoltà principale consiste nel trovare gli integrali purticolari. Noi abbiamo aftrontato il problenn ponendori is un caso partirolare, cioè assumendo a priori usa relazioute lineare fra i coeflicienti elastici al secondo ordine senza ridure signifiativamente il campo della loro variabilità e quindi la possibilità di una eflettiva applicazione al modello elastico della Terra. Una teoria più rompleta potrà contemplàre anche la possibilità di risolvere tali equazioui in un molo meno restrittivo eventualnente mediaute metorli mumerici, roi quali però si perderebbe parte della capacità di romprensione diretta fisica del festomeno, che il metodo qui seguito ei hat permesso.

\section{BIBT,1OGRAG]A}

(1) CaLor P., Sulle oseillazioni libere della terra edpl suo qusoio esterno, Ace. Naz. Jincei, Serie VIII, XXXII, 4, (1962).

(2) Bozzi-Zapho H., Caputo M., Spectral, Bispectral Analysis and $Q$ of the free oscillations of the Farth, "Suppl. Nuovo Cimento n, Serie I, 6, pag. 67-81, (1908).

(9) Caputo M., Elasticitì e dissipazione, Zanicholli, Bolognat (1969).

(4) Caputo M., Rossi-Tesi F., Lo apetfo delle oseillazioni libere della Terra, "Suppl. Nuovo Cimento ", Serie I, 6, pag. 857-865, (1968).

(5) Munkagitan F. D., Finite deformation of an elastic solid, fohn Wiley \& Sms, luc., New York, (I95I). 